\title{
ANEURISMA GIGANTE DA ARTÉRIA CEREBRAL MÉDIA
}

\author{
J. JoRge FACURE* \\ NUBOR O. FACURE ** \\ LuIZ F. CASTRO *** \\ JoAquim N. CRUZ***
}

Registramos a ocorrência de aneurisma gigante da artéria cerebral média em uma paciente com manifestações clínicas semelhantes as dos tumores cerebrais. Os aneurismas gigantes constituem patologia rara nas artérias encefálicas, se manifestam como processo expansivo e dificilmente apresentam hemorragia meníngea $4,6,7,8,9$.

\section{O B S E R V A Ç A}

A.M.F., brasileira, branca, 28 anos, sexo feminino, admitida em 4-8-73 com sindrome de hipertensão intracraniana, com 5 meses de evolução. Os primeiros sintomas apresentados pela paciente foram constituidos por crises com sensação súbita de mau odor e formigamento no membro superior direito acompanhados de automatismo mastigatório e salivação. Estas crises foram de imediato controladas com anticonvulsivantes. Nesta ocasião foi feito eletrencefalograma (EEG) que foi normal. A paciente apresentava desde o início cefaléia difusa, pulsátil, contínua, com pequenos períodos de acalmia. Uma semana antes da internação sofreu perda súbita da consciência. Após a crise apresentou cefaléia e sonolência, não referindo manifestações prodrômicas. Houve recuperação progressiva permanecendo apenas cefaléia. Estudo eletrencefalográfico realizado nesta época mostrou assimetria, com sofrimento frontotemporal esquerdo. Exame clinico-neurológico - Sindrome de hipertensão intracraniana com papiledema bilateral sem sinais neurológicos focais. Exame neurorradiológico - A angiografia cerebral carotídea esquerda mostrou neurisma gigante ao nível da artéria cerebral média (Fig. 1). Completamos o estudo angiográfico encefálico por cateterismo da artéria braquial direita, tendo sido afastada a ocorrência de outras malformacões vasculares nos territórios vértebro-basilar e carotídeo direito.

Intervenção cirúrgica - Em 8-8-73 foi feita cranitomia frontotemporal esquerda. Realizamos corticotomia ao nivel do giro temporal superior, tendo sído encontrada a parede do aneurisma a $10 \mathrm{~mm}$ de profundidade. A malformação que apresentava parede espessa, foi totalmente dissecada e, após ligadura do colo com clipe de Heifetz, foi extirpada.

Exame histopatológico - Trata-se de formação irregularmente prismática, encapsulada, castanho amarelada medindo $35 \mathrm{~mm}$ de base por $27 \mathrm{~mm}$ de altura $\times 23 \mathrm{~mm}$ de espessura. Fixação em formol, inclusão em parafina e coloração por hematoxilinaeosina, van Gieson e orceina Giemsa (OG). Ao exame microscópico encontra-se

Trabalho do Departamento de Neurologia da Faculdade de Ciências Médicas da UNICAMP: * Neurocirurgião; ** Coordenador do Departamento; *** Médicos residentes. 


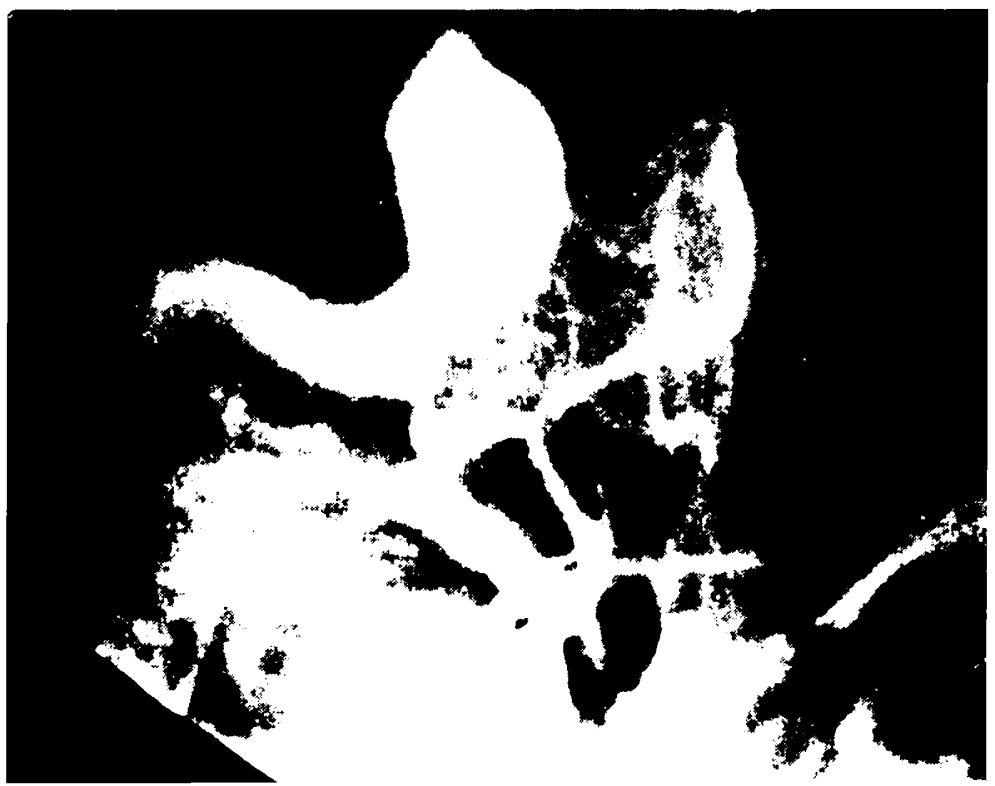

Fig. 1 - Caso A.M.F. Arteriografia carotidca esquerda: aneurisma gigante da artéria cerebral média.

parede arterial reconhecivel na periferia, representada por uma faixa de tecido colágeno fibrilar. Focos de grânulos calcificados podem ser encontrados nas áreas mais espessas geralmente no centro da faixa. A face interna fica em contato direto com material hialino acidófilo com restos de sangue. A lâmina elástica foi demonstrada com a coloracão pela OG. Classificação: aneurisma com trombo em organização (Fig. 2).

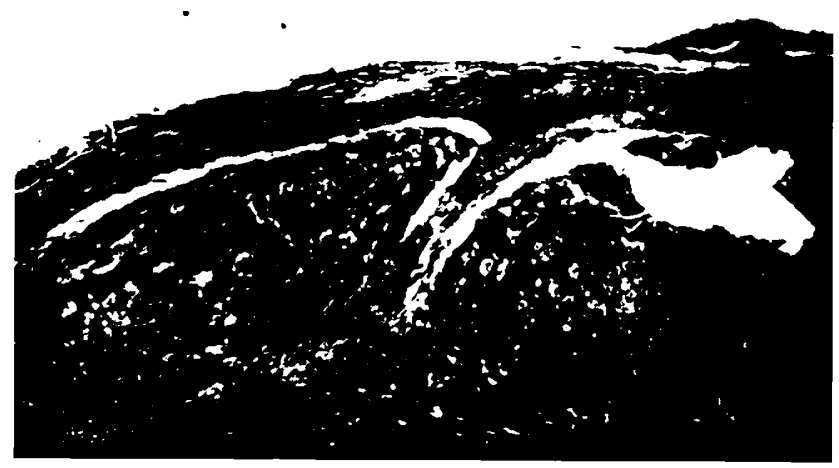

Fig. 2-Caso A.M.F. Trombo dissecando a parede do aneurisma (O.G., $\times 50$ ). 
Evolução - Melhora progressiva com desaparecimento dos sintomas de hipertensão intracraniana. Não ocorreu deficit motor no pós-operatório. Sete dias após a cirurgia realizamos estudo angiográfico tendo sido demonstrado que a exerese do aneurisma não determinou comprometimento vascular (Fig. 3). Com 30 dias de seguimento, a paciente apresenta-se bem não havendo manifestaçōes subjetivas. $O$ exame clínico-neurológico revelou apenas papilas ópticas com limites imprecisos traduzindo papiledema pregresso.

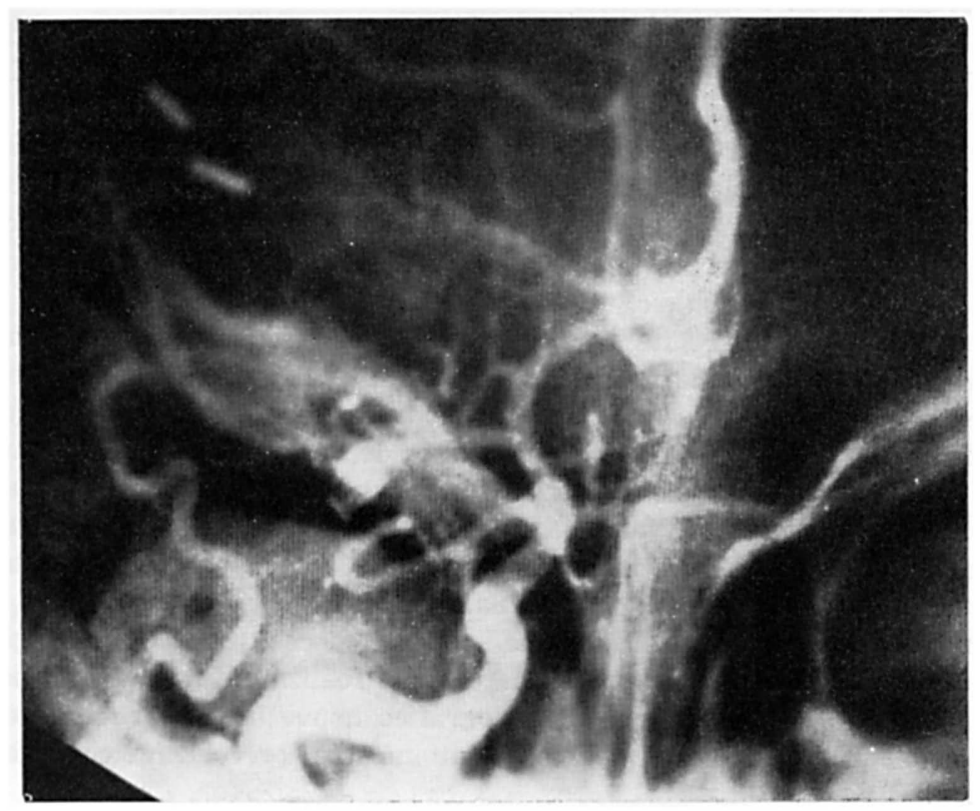

\footnotetext{
Fig. 3 - Caso A.M.F. Arteriografia carotidea esquerda no $7^{\circ}$ dia de pós-operatório: não houve comprometimento da artéria cerebral média.
}

\section{O M E N T Á R I O S}

Morley e Barr ${ }^{5}$ consideram como aneurisma gigante o que apresenta diâmetro maior do que $25 \mathrm{~mm}$. Fazem referência a 17 casos, três dos quais da artéria cerebral média.

Stehbens ${ }^{8}$ com uma casuística de 333 aneurismas cita 19 casos com diâmetro maior do que $20 \mathrm{~mm}$, sendo que 5 pacientes apresentaram quadro clínico simulando tumor intracraniano. $O$ autor salienta que estes aneurismas podem ocorrer na artéria vertebral, na artéria basilar, na carótida interna, na artéria cerebral média ou na região da artéria comunicante anterior. Dependendo de sua localização podem ocorrer manifestações clínicas semelhantes às dos adenomas da hipófise, a tumor cerebral, a tumor de fossa posterior incluindo-se tumor do ângulo ponto-cerebelar ${ }^{3}$. 
Adams ${ }^{1}$ admite que o aneurisma gigante seja uma malformação congênita que assume proporções volumosas já desde o início. Reforçando esta opinião a literatura registra sua incidência na infância ${ }^{4}$.

Sadik e col. ${ }^{6}$ relataram um caso de aneurisma da artéria cerebral média medindo $85 \times 55 \times 50 \mathrm{~mm}$ que se manifestou como processo expansivo em uma paciente de 47 anos de idade: chamam a atenção para a informação de comprometimento visual desde a infância, o que poderia sugerir a existência do aneurisma desde o nascimento.

Cuatico e col. ${ }^{2}$ documentaram dois casos de aneurismas gigantes que se desenvolveram a partir de aneurismas menores, da porção supraclinoidea da carótida. Nos dois pacientes, os aneurismas, quando apresentavam ainda pequeno volume, foram tratados por ligadura da carótida na região cervical. Com seqüência angiográfica de três anos foi confirmado o aumento progressivo do volume dos aneurismas. Os autores admitem ter havido coagulação parcial do aneurisma, a porção coagulada se constituiria em "locus minoris resistentia", facilitando a sua dilatação.

Terao e Muraoka ${ }^{9}$ relatando um caso de volumoso aneurisma da artéria cerebral média, medindo $80 \times 55 \times 60 \mathrm{~mm}$, admitem também que o aneurisma gigante pode aumentar o seu volume à custa de hemorragias intramurais ou intratrombóticas com conseqüente processo de organização. Estes autores preconizam a extirpação do aneurisma para aliviar a hipertensão intracraniana e, mesmo, para prevenir eventual hemorragia. Salientam também que a cirurgia às vezes não é possível de ser executada sem comprometimento da artéria da qual o aneurisma se origina.

Por outro lado, Scott e Ballantine ${ }^{7}$ citam um caso de aneurisma gigante assintomático da artéria cerebral média em que realizaram seguimento neurorradiológico por 5 anos. Ao estudo angiográfico houve desaparecimento expontâneo do aneurisma. Para os casos assintomáticos, preconizam conduta conservadora.

O caso por nós apresentado sugere que houve crescimento progressivo do aneurisma. Por ocasião da primeira consulta o exame clínico-neurológico e o EEG foram normais. Com intervalo de 5 meses comprovamos papiledema e EEG assimétrico, com sofrimento cerebral frontotemporal esquerdo.

\section{R E S U M O}

Relato de um caso de aneurisma gigante da artéria cerebral média esquerda operado com sucesso. $O$ aneurisma que apresentava medidas de $35 \times 27 \times 23 \mathrm{~mm}$ determinou manifestações clínicas de hipertensão intracraniana. A sua exerese total resultou em pronto desaparecimento dos sintomas.

\section{S U M M A R Y}

Giant aneurysm of the middle cerebral artery: a case report

A case of giant aneurysm of the left middle cerebral artery, in a 28-year-old right-handed woman, successfuly operated is reported. The aneurysm measur- 
ing $35 \times 27 \times 23 \mathrm{~mm}$ showed clinical manifestations as a space-occupying lesion. The patient recovered completely after the total excision of the aneurysm.

\section{REFERENCIAS}

1. ADAMS, R. D. - Case records of the Massachusetts General Hospital. Case 22-1963. New Engl. J. Med. 268:724, 1963.

2. CUATICO, W.; COOK, A. W.; TYSHCHENKO, V. \& KHATIB, R. - Massive enlargement of intracranial aneurysms following carotid ligation. Arch. Neurol. (Chicago) 17:609, 1967.

3. HAREL, D.; LAVY, S. \& SCHWARTZ, A. - Aneurysm of basilar artery simulating a cerebellopontine angle tumor. Confin. neurol. 29:360, 1967.

4. JANE, J. A. - A large aneurysm of the posterior inferior cerebellar artery in a 1-year-old child. J. Neurosurg. 18:245, 1961.

5. MORLEY, T. P. \& BARR, H. W. K. - Giant intracranial aneurysms: diagnosis, course and management. Clin. Neurosurg. 16:73, 1968.

6. SADIK, A. R.; BUDZILOVICH, G. N. \& SHULMAN, K. - Giant aneurysm of middle cerebral artery. J. Neurosurg. 22:177, 1965.

7. SCOTT, R. M. \& BALLANTINE Jr., H. T. - Spontaneous thrombosis in a giant middle cerebral artery aneurysm. J. Neurosurg. 37:361, 1972.

8. STEHBENS, W. E. - Intracranial arterial aneurysms. In Stehbens, W. E. Pathology of the Cerebral Blood Vessels. C. V. Mosby Co., Saint Louis, 1972, pp. 351-470.

9. TERAO, H. \& MURAOKA, I. - Giant aneurysm of the middle cerebral artery containing an important blood channel. J. Neurosurg. 37:352, 1972.

Clinica Neurológica - Faculdade de Ciências Médicas - Caixa Postal 1170 19100 Campinas, SP - Brasil. 